\title{
The impact of chronic disease self-management programs: healthcare savings through a community-based intervention
}

\author{
SangNam Ahn ${ }^{1,7^{*}}$, Rashmita Basu' ${ }^{2}$, Matthew Lee Smith ${ }^{3,7}$, Luohua Jiang ${ }^{4}$, Kate Lorig ${ }^{5}$, Nancy Whitelaw ${ }^{6}$
} and Marcia G Ory ${ }^{7}$

\begin{abstract}
Background: Among the most studied evidence-based programs, the Chronic Disease Self-Management Program (CDSMP) has been shown to help participants improve their health behaviors, health outcomes, and reduce healthcare utilization. However, there is a lack of information on how CDSMP, when nationally disseminated, impacts healthcare utilization and averts healthcare costs. The purposes of this study were to: 1) document reductions in healthcare utilization among national CDSMP participants; 2) calculate potential cost savings associated with emergency room (ER) visits and hospitalizations; and 3) extrapolate the cost savings estimation to the American adults.

Methods: The national study of CDSMP surveyed 1,170 community-dwelling CDSMP participants at baseline, 6 months, and 12 months from 22 organizations in 17 states. The procedure used to estimate potential cost savings included: 1) examining the pattern of healthcare utilization among CDSMP participants from self-reported healthcare utilization assessed at baseline, 6 months, and 12 months; 2) calculating age-adjusted average costs for persons using the 2010 Medical Expenditure Panel Survey; 3) calculating costs saved from reductions in healthcare utilization; 4) estimating per participant program costs; 5) computing potential cost savings by deducting program costs from estimated healthcare savings; and 6) extrapolating savings to national populations using Census data combined with national health statistics.
\end{abstract}

Results: Findings from analyses showed significant reductions in ER visits (5\%) at both the 6-month and 12 -month assessments as well as hospitalizations (3\%) at 6 months among national CDSMP participants. This equates to potential net savings of $\$ 364$ per participant and a national savings of $\$ 3.3$ billion if $5 \%$ of adults with one or more chronic conditions were reached.

Conclusions: Findings emphasize the value of public health tertiary prevention interventions and the need for policies to support widespread adoption of CDSMP.

Keywords: Chronic disease self-management program, Healthcare utilization, Healthcare cost savings

\footnotetext{
* Correspondence: sahn@memphis.edu

'School of Public Health, Division of Health Systems Management and Policy,

The University of Memphis, Memphis, TN, USA

${ }^{7}$ Department of Health Promotion and Community Health Sciences, School

of Rural Public Health, Texas A\&M Health Science Center, College Station, TX,

USA

Full list of author information is available at the end of the article
} 


\section{Background}

People living with prevalent chronic conditions such as heart disease, cancer, diabetes, stroke, and chronic lung disease account for $75 \%$ of healthcare expenditures in the United States [1]. This disease burden profile will exacerbate with the rapid aging of the American population. The number of Americans with chronic conditions is projected to increase by $37 \%$ (i.e., 46 million people) from 2000 to 2030 [2].

The Patient Accountability and Affordable Care Act underscores the potential of chronic disease management for improving the efficiency of healthcare in the U.S. This Act also recognizes the importance of community. Helping people develop the knowledge, skills, and motivation needed to make healthier choices for better self-management is seen as an essential part of the national prevention strategy [3]. Toward this end, there is growing evidence that structured small group chronic disease self-management education, such as Stanford's Chronic Disease Self-Management Program (CDSMP), can help participants improve their health behaviors, health outcomes, and reduce healthcare utilization $[4,5]$. Although it has been more than 10 years since positive findings from the initial randomized trial were reported, there is no current information on how healthcare utilization and associated healthcare costs can be averted by a nationwide dissemination of CDSMP.

Thus, the current study examines reductions in healthcare utilization among CDSMP participants to identify potential cost savings to the health system as a result of participating in the program. Three specific purposes of this study were to: 1) document reductions in healthcare utilization among participants of the national study of CDSMP; 2) calculate potential cost savings associated with emergency room (ER) visits and hospitalizations using age-adjusted national cost estimates; and 3) extrapolate the cost savings estimation to the American adults with one or more chronic conditions. Drawing upon findings from a parallel nationwide CDSMP study [6], the current study proposes to inform policy makers, state officials, healthcare providers, and community agencies about potential healthcare cost savings.

\section{Methods}

CDSMP is an evidence-based, peer-led program consisting of six sessions hosted over six consecutive weeks that empowers participants to develop skills necessary for medical, social role, and emotional management of chronic conditions [4]. It is a public health intervention delivered by trained facilitators in community-based settings throughout the U.S. and around the globe. CDSMP workshops are supported by various federal, state, and local sources as well as healthcare organizations and community agencies. As a translational research study, the National Study of CDSMP surveyed 1,170 communitydwelling CDSMP participants at baseline, 6 months, and 12 months from 22 organizations in 17 states. Approximately $77 \%(n=903)$ and $71 \%(n=825)$ of the 1,170 participants completed the 6-month and 12-month assessments, respectively [6,7]. Participants who completed assessments at both time points tended to be older, and completers of the 6-month assessment were more likely to be nonHispanic white [6]. Self-reported data using validated questionnaires were collected about health conditions, health behaviors, and healthcare utilization from a multi-ethnic population (55.2\% were non-Hispanic white) [8].

The procedure used to estimate potential cost savings started by examining the pattern of healthcare utilization among CDSMP participants from self-reported healthcare utilization (i.e., ER visits and hospitalizations) assessed at baseline, 6 months, and 12 months. Generalized mixed effects models (using Stata gllamm procedure; [9]) were used to assess change for any ER visits and hospitalizations (binary) from baseline to 6- and 12-month assessments controlling for age, sex, race/ethnicity, education, and number of chronic conditions. Demographic statistics and coding schemes for these variables are documented previous studies [6,7]. These mixed effects models used likelihood-based approaches to provide unbiased estimates of the intervention effects assuming that responses are missing at random.

Once the patterns of health care were documented, the following procedure were undertaken to examine cost issues: 1) calculating age-adjusted average costs for persons with at least one chronic condition using weighted average costs (using MEPS sampling weights) for ER visits and hospitalizations from the Household Component of the 2010 Medical Expenditure Panel Survey (MEPS) [10]; 2) calculating costs saved from reductions in healthcare utilization adjusting for population distribution for three age groups (18-44, 45-64, and 65+); 3) estimating per participant program costs based on expert opinion from program developers and budget calculations from agencies offering the program; 4) computing potential cost savings by deducting program costs from estimated healthcare savings; and 5) extrapolating savings to national populations using Census data [11] combined with national health statistics. Institutional Review Board approval was obtained through Texas A\&M University.

\section{Results}

Table 1 shows changes in ER visits from baseline (18\%) to 6 months (13\%) to 12 months (13\%). The odds of ER visits in the past 6 months among CDSMP participants was significantly reduced from baseline to 6-month (Odds Ratio $[\mathrm{OR}]=0.68, \mathrm{p}=0.007)$ and 12-month $(\mathrm{OR}=0.68, \mathrm{p}=$ 0.009 ) assessments controlling for potential confounding 
Table 1 Adjusted ${ }^{\mathrm{a}}$ ratios between baseline and follow-up means for ER visits and Hospitalizations among CDSMP participants $(\mathrm{N}=1,170)$

\begin{tabular}{|c|c|c|c|c|c|c|c|}
\hline & \multicolumn{3}{|l|}{$\%^{b}$} & \multicolumn{2}{|c|}{$\begin{array}{l}\text { Adjusted }^{c} \text { change from } \\
\text { baseline to 6-month }\end{array}$} & \multicolumn{2}{|c|}{$\begin{array}{l}\text { Adjusted change }{ }^{d} \text { from } \\
\text { baseline to } 12 \text {-month }\end{array}$} \\
\hline & $\begin{array}{l}\text { Baseline } \\
(n=1,170)\end{array}$ & $\begin{array}{l}\text { 6-Month } \\
(\mathrm{n}=903)\end{array}$ & $\begin{array}{l}\text { 12-month } \\
(\mathrm{n}=825)\end{array}$ & $\begin{array}{l}\text { Adjusted ratio } \\
\text { change }\end{array}$ & P-value & $\begin{array}{l}\text { Adjusted ratio } \\
\text { change }\end{array}$ & P-value \\
\hline Any ER visit & $18 \%$ & $13 \%$ & $13 \%$ & 0.68 & 0.007 & 0.68 & 0.009 \\
\hline Any hospitalization & $14 \%$ & $11 \%$ & $14 \%$ & 0.70 & 0.025 & 1.02 & 0.920 \\
\hline
\end{tabular}

${ }^{a}$ All changes are adjusted for sex, age, race/ethnicity, education, and number of chronic conditions.

${ }^{\mathrm{b}}$ Raw percentage at baseline, 6-month, and 12-month.

${ }^{c}$ Adjusted odds ratio of any ER visit and hospitalization between baseline and 6-month from the logistic regression models.

${ }^{\mathrm{d}}$ Adjusted odds ratio of any ER visit and hospitalization between baseline and 12-month from the logistic regression models.

factors. Table 1 also shows changes in hospitalizations from baseline (14\%) to 6 months $(11 \%)$ to 12 months (14\%). The adjusted odds of hospitalizations was significantly reduced from baseline to 6-month assessment $(\mathrm{OR}=0.70, \mathrm{p}=0.025)$.

Table 2 describes calculated cost savings associated with ER visits and hospitalizations among the national study of CDSMP participants. As shown in Table 1, significant reductions in ER use were observed at both the 6-month and 12-month assessments (5\% at each time point). There was a significant reduction for hospitalizations at 6 months (3\%). The estimated average cost for ER visits and hospitalizations of those having at least one chronic condition from 2010 MEPS data were $\$ 1,513$ and $\$ 18,750$, respectively. Total estimated health care costs averted per participant were calculated as $\$ 713.80$.

Table 2 also displays variation in the average per participant cost of CDSMP by number of enrolled participants per workshop. Assuming a \$3,500 per workshop cost, the estimated per participant cost ranged from $\$ 583.33$ (i.e., 6 participants) to $\$ 350$ (i.e., 10 participants) to $\$ 218.75$ (i.e., 16 participants). In a previous national study of 145 CDSMP workshops, the workshops had an average size of $12.7( \pm 4.18)$, with the majority of workshops $(66.2 \%)$ having between 8 and 16 participants [12]. There were small extremes at both ends with $17.9 \%$ of the workshops having less than 8 participants and $15.9 \%$ having more than 16 participants [12]. When assuming $\$ 350$ per participant cost based on best estimates from experts and field reports, potential cost savings were estimated to be $\$ 363.80$ per person (i.e., $\$ 713.80-\$ 350.00$ ). Extrapolating these savings to the national level, potential savings of $\$ 65.7$ billion could be achieved if CDSMP reached all individuals with one or more chronic condition. More feasibly, potential savings of $\$ 3.3$ billion could be achieved if the program reached only $5 \%$ of this population (approximately 9 million people) or $\$ 0.7$ billion reaching $1 \%$ of this population (approximately 1.8 million people). When assuming \$218.75 per participant cost, national healthcare savings were estimated $\$ 8.9$ billion reaching $10 \%$ of this population, $\$ 4.5$ billion reaching $5 \%$, and 0.9 billion reaching $1 \%$. If assuming $\$ 583.33$ per participant cost, national healthcare savings were estimated at $\$ 2.4$ billion if the program reached $10 \%$ of this population, $\$ 1.2$ billion reaching $5 \%$, and $\$ 0.2$ billion reaching $1 \%$.

\section{Discussion}

This study reaffirms the importance and potential of community-based self-management interventions rooted in public health to control healthcare costs among adults with chronic conditions. Extrapolating the estimated \$364 cost saving per CDSMP participant results in meaningful national savings (i.e., $\sim \$ 3.3$ billion), if program penetration reaches only $5 \%$ of all individuals with one or more chronic condition. Assuming a \$350 average CDSMP cost per participant, we could achieve potential national healthcare savings from $\$ 0.7$ billion to $\$ 65.7$ billion by averting from ER visits and hospitalizations if CDSMP reaches a minimum of $1 \%$ of adults having at least 1 chronic condition (i.e., the range of national savings depends on the level of program penetration). The cost savings achieved herein among heterogeneous populations served by diverse organizations were substantial; however, they were slightly lower than those estimated in the original, more tightly controlled, randomized trial which included controls whose hospital-related healthcare costs increased [4].

Previous studies have documented the value of CDSMP in improving participants' health behaviors, diseaserelated symptoms, communications with providers, and overall health status $[4,5]$. With the addition of findings from the current study, it is clear that this intervention can influence all aspects of the Triple Aim (i.e., enhanced care, improved health, and better value) $[6,13]$. Modest past investments by the U.S. Administration on Aging, the Centers for Disease Control and Prevention, and other agencies have established a viable foundation for scaling up this intervention. Within the past 5 years, over 150,000 people have participated in CDSMP workshops through the Communities Putting Prevention to Work Initiative and other public-private collaborations [12]. This highlights the probability of high-level CDSMP penetration to reach populations with chronic conditions as long as strong support and funding sources are available for this initiative. As such, additional public sector resources are 
Table 2 Cost savings estimation based on 2010 national CDSMP data and 2010 Medical Expenditure Panel Survey (MEPS)

Potential cost savings related to ER visits and hospitalizations among CDSMP participants

Potential annual health care savings per CDSMP participant (A)

$\$ 713.80$

Age-adjusted cost of ER visits among those having at least 1 chronic condition (CC) $\$ 1,513$

(Baseline $=18 \% ; 6$ month post $=13 \%)$

(Baseline $=18 \% ; 12$ month post $=13 \%$ )

5\% Reduction of ER visits among CDSMP participants in the 1st 6-months

$\$ 75.65$

Age-adjusted cost of hospitalizations among those having at least $1 \mathrm{CC}^{\mathrm{a}}$

$5 \%$ Reduction of ER visits among CDSMP participants in the 2nd 6-months

(Baseline $=14 \% ; 6$ month post $=11 \%$ ) $\$ 18,750$

(Baseline $=14 \% ; 12$ month post $=14 \%$

$3 \%$ Reduction of hospitalizations among CDSMP participants in the 1st 6-months $\$ 562.50$

$0 \%$ Reduction of hospitalizations among CDSMP participants in the 2nd 6-months \$0.00

Estimated number of CDSMP participants (B)

Estimated CDSMP workshop cost ${ }^{\mathrm{b}}(\mathrm{C})$

Estimated average CDSMP costs per person varying by number of CDSMP participants and workshop costs $(D=C \div B)^{b}$

Net cost savings per CDSMP participant $(E=A-D)$

\section{Extrapolation to national savings using Census data combined with MEPS data}

Number of Americans aged 18 and older from 2010 Census data (F)

Estimated \% of Americans having at least $1 \mathrm{CC}^{\mathrm{a}}(\mathrm{G})$

Number of Americans aged 18 and older having at least $1 \mathrm{CC}(\mathrm{H}=\mathrm{F} \times \mathrm{G})$

$234,564,071$

$77 \%$

$180,614,335$

Estimated number of CDSMP participants

$\$ 3,500.00 \$ 3,500.00$

$\$ 583.33 \$ 350.00 \quad \$ 218.75$

$\$ 130.47 \$ 363.80 \quad \$ 495.05$

National health care savings if we could reach

$100 \%$ of people having at least $1 \mathrm{CC}(\mathrm{E} \times \mathrm{H})$

$10 \%$

$5 \%$

lars

$\$ 23.6 \quad \$ 65.7 \quad \$ 89.4$

$\$ 2.4 \quad \$ 6.6 \quad \$ 8.9$

$\$ 1.2 \$ 3.3 \quad \$ 4.5$

$\$ 0.2 \quad \$ 0.7 \quad \$ 0.9$

Note. ${ }^{\mathrm{a}}$ Based $2010 \mathrm{MEPS} ;{ }^{\mathrm{b}}$ Based on reported data from two CDSMP national studies in the states of Oregon and Florida and expert's opinions including CDSMP developers. 
needed to continue the momentum and leverage the existing infrastructure. At the same time, private insurers are highly encouraged to provide benefits to their patients with chronic conditions by discounting their premiums as well as CDSMP workshop delivery agencies by providing generous reimbursements.

A policy issue of interest surrounds how efficiencies in CDSMP workshop delivery can increase overall savings. In the current study, we have used an estimated $\$ 350$ per participant cost in our calculations assuming 10 participants in a workshop and $\$ 3,500$ workshop costs. To estimate this average cost, we relied on experts' opinions including the program developers, field reports (ranging from \$204 to \$375) [14,15], and an unpublished survey among state CDSMP implementers conducted by the National Council on Aging (ranging from \$150 to \$750) [16]. However, it is worth noting that the cost estimation should vary by the number of CDSMP participants in a workshop and the administrative capacity of the delivering agencies. Nevertheless, we would expect costs to decrease with the efficiencies gained through capacity building accompanying widespread dissemination. A prior study projected lower average CDSMP costs for agencies with higher numbers of participants over time compared to the other agencies with lower numbers of participants, which emphasizes the importance of strong recruitment efforts and collaborating with community partners [15]. Further studies are warranted to identify how costs associated with marketing and administration would be affected when scaling up for widespread program delivery.

It is important to ensure that the cost-saving benefits of CDSMP equitably reach various populations despite geographic location and demographic factors. The small enrollment in some workshops highlights potential difficulties of scaling up in rural areas as these areas typically have smaller class sizes due to population dispersion and lack of infrastructure supports. However, on the positive side, CDSMP is being widely disseminated throughout the United States (e.g., between 2010 and 2012 more than 100,000 participants enrolled in CDSMP programs sponsored by the Administration on Aging) [12]. A prior study analyzing demographic factors and disease profiles among more than 100,000 CDSMP participants (between 2010 and 2012) documented how representative CDSMP participants are of the adult population [12]. When comparing to 2010 U.S. Census, CDSMP participants tended to be more female $(77.7 \%)$ and older (mean age $=67$ years) compared to the Census (51\%, 37 years) [11]. However, there were similarities in terms of rural residence and race/ethnicity. Approximately 25\% of CDSMP participants resided in rural areas (compared to $19.3 \%$ of Census) and had a similar racial/ethnic composition (white of CDSMP: $66.4 \%$ vs. $63.7 \%$ of Census; African American: 21.5\% vs. 12.2\%; Hispanic: $17.0 \%$ vs. $16.3 \%$; Asian/Pacific Islanders: $4.5 \%$ vs. $4.9 \%$;
American Indians: $1.6 \%$ vs. 0.7\%) [17]. These statistics are encouraging, especially when considering the capacity of evidence-based interventions to reach various populations at risk of chronic conditions.

Additionally, the potential of CDSMP to contribute to cost savings while improving health status provides a strong incentive for alignment with Accountable Care Organizations, models of enhanced primary care, initiatives for dually eligible beneficiaries, and State Innovation Models. To better integrate and leverage CDSMP to improve healthcare organization and financing, new initiatives are needed to design and test ways to: 1) strengthen collaboration among healthcare organizations, community partners, and public health agencies; 2) establish useful quality measures related to self-management; and 3) incentivize providers to further support evidence-based approaches to self-management.

\section{Study limitations}

First, data were drawn from a national study with a prepost design appropriate for addressing translational research questions. While the current study lacks a comparison group, improvements were generally similar as those reported in the original randomized trial with some attenuated cases [4]. Our current study design does not permit the elimination of potentially confounding factors influencing study outcomes. Second, healthcare utilization was self-reported resulting in the possibility of recall bias. Nevertheless, a prior study found high concordance between self-reported and objectively measured ER visits and inpatient use [18]. Third, the current 12-month study may require a longer study duration to conclude definitively the healthcare cost-saving effects of CDSMP; however, we expect to see sustainable effects of reducing ER visits based on prior 2-year study [4]. Last, the current study is based on a critical assumption that we can extrapolate the healthcare cost savings of the National CDSMP to the national level using census data. Therefore, the costsaving effects of CDSMP should be further studied to account for demographic changes in the Americans population over time and variations by population's disease profiles. Nevertheless, we caution that it will be difficult to accurately estimate cost-saving effects by specific chronic condition types given the presence of multiple chronic conditions and the multitude of different disease clusters. To provide context, a previous study reported that CDSMP participants have on average 2.2 chronic conditions (e.g., hypertension $=43.0 \%$; arthritis $=40.8 \%$; diabetes $=30.3 \%$; depression $=19.5 \%$ ) [12]. Future study is needed to examine the average per participant cost of CDSMP based on geographic locations and capacities of agencies to deliver CDSMP. 


\section{Conclusion}

Future efforts should explore these issues in light of the complexities of estimating costs and cost savings from existing field studies. However, the fundamental findings of the current study (i.e., the potential of CDSMP type programs to accrue cost savings through decreased ER visits and hospitalizations) suggest that evidence-based self-management programs are cost-saving and health-enhancing strategies for dealing with the epidemic of chronic conditions, especially with workshops with 10 or more participants. We recommend immediate attention be given to initiate system changes and policies that increase the awareness of selfmanagement programs among patients as well as physicians, support the development of a delivery infrastructure, and help defray the costs of widespread dissemination of such programs. CDSMP delivers wide range of important outcomes, with a return on investment of $1: 1$ - which means this tertiary prevention intervention provides substantial value, more than paying for itself.

\section{Abbreviations}

CDSMP: Chronic disease self-management program; ER: Emergency room; MEPS: Medical expenditure panel survey.

\section{Competing interests}

The authors declare that they have no competing interests, with one exception that we are disclosing. Kate Lorig, the program developer, receives royalties from the book used by participants in the CDSMP program. However, independent analyses were conducted on all study outcomes by Texas A\&M Health Science Center.

\section{Authors' contributions}

SA planned the study, analyzed the data, and wrote the article. RB analyzed the data and wrote the article. MLS assisted in data interpretation and in critical revision. $L J$ analyzed the data. $\mathrm{KL}$ assisted in data interpretation and provided critical revisions. NW provided critical revisions. MGO conceived and supervised the study, and provided critical content and revisions. All authors read and approved the final manuscript.

\section{Acknowledgements}

We recognize grant support from the Administration on Aging through American Recovery and Reinvestment Act funding for the Chronic Disease SelfManagement Program. This work was supported by the National Council on Aging (NCOA) through contracts to Texas A\&M Health Science Center (Principal Investigator: Marcia Ory) and Stanford University (Principal Investigator: Kate Lorig). Nancy Whitelaw served as the NCOA Principal Investigator. We thank the 22 delivery sites and the participants who enrolled in the National Study of Chronic Disease Self-Management Program from 2010-2011. Diana Laurent, Audrey Alonis, Maurice Green, Eileen Bradley, Angela Devlin, and Phillip Ritter from Stanford Patient Education Research Center assisted in the data collection and management. Additionally, funding was made possible (in part) from the Centers for Disease Control and Prevention cooperative agreements to Prevention Research Centers Programs in support of the Healthy Aging Research Network (1U48/DP001924). We also acknowledge support from NIH (1RC4AG038183-01) for funding of the Community Research Center on Senior Health.

\section{Author details}

'School of Public Health, Division of Health Systems Management and Policy, The University of Memphis, Memphis, TN, USA. ${ }^{2}$ Scott \& White Healthcare, Temple, TX, USA. ${ }^{3}$ Department of Health Promotion and Behavior, College of Public Health, The University of Georgia, Athens, GA, USA. ${ }^{4}$ Department of Epidemiology and Biostatistics, School of Rural Public Health, Texas A\&M Health Science Center, College Station, TX, USA. ${ }^{5}$ Department of Medicine, Stanford University, Palo Alto, CA, USA. ${ }^{6}$ National Council on Aging,
Washington, DC, USA. ${ }^{7}$ Department of Health Promotion and Community Health Sciences, School of Rural Public Health, Texas A\&M Health Science Center, College Station, TX, USA.

Received: 15 July 2013 Accepted: 8 November 2013

Published: 6 December 2013

\section{References}

1. Harris JR, Wallace RB: The institute of Medicine's New report on living well with chronic illness. Prev Chronic Dis 2012, 9:E148. DOl: 10.5888/pcd9.120126.

2. Anderson GF: Chronic care: making the case for ongoing care. Princeton, NJ: Robert Wood Johnson Foundation; 2010.

3. National Prevention Council: National prevention strategy. Washington, DC: US Department of Health and Human Services, Office of the Surgeon General; 2011.

4. Lorig KR, Ritter P, Stewart AL, Sobel DS, Brown BW Jr, Bandura A, Gonzalez VM, Laurent DD, Holman HR: Chronic disease self-management program: 2-year health status and health care utilization outcomes. Med Care 2001, 39(11):1217-1223.

5. Brady TJ, Murphy L, Beauchesne D, Bhalakia A, Chervin D, Daniels B, Greenberg M, House M, O'Colmain B: Sorting through the evidence for the Arthritis Self-Management Program and the Chronic Disease Self-Management Program: Executive Summary of ASMP/CDSMP metaanalysis. Atlanta: Centers for Disease Control and Prevention; 2011. http:// www.cdc.gov/arthritis/docs/asmp-executive-summary.pdf.

6. Ory MG, Ahn S, Jiang L, Smith ML, Ritter P, Whitelaw N, Lorig K: Successes of a national study of the chronic disease self-management program: meeting the triple Aim of health care reform. Med Care 2013, 51(11):992-998.

7. Ory MG, Ahn S, Jiang L, Lorig K, Ritter P, Laurent D, Whitelaw N, Smith ML: National study of chronic disease self-management: six month outcome findings. J Aging Health 2013, 25(7):1258-1274.

8. Whitelaw N, Lorig K, Smith ML, Ory MG: National Study of Chronic Disease Self-Management Programs (CDSMP). 2013. from www.ncoa.org/cha.

9. Lee $A H$, Zhao $Y$, Yau KKW, Xiang L: How to analyze longitudinal multilevel physical activity data with many zeros? Prev Med 2010, 51(6):476-481.

10. Agency for Health Research and Quality (AHRQ): Medical Expenditure Panel Survey Household Component: 2010 Full Year Consolidated Data File. Rockville, MD; 2010. http://meps.ahrq.gov/mepsweb/data_stats/ download_data_files_detail.jsp?cboPufNumber=HC-138.

11. Howden LM, Meyer JA: Age and sex composition: 2010. In 2010 Census Briefs, US Department of Commerce, Economics and Statistics Administration US Census Bureau. Washington, DC; 2010. Retrieved May 10, 2013, from http://www.census.gov/prod/cen2010/briefs/c2010br-03.pdf.

12. Ory MG, Smith ML, Patton K, Lorig K, Zenker W, Whitelaw N: Self-management at the tipping point: reaching 100,000 Americans with evidence-based programs. J Am Geriatr Soc 2013, 61(5):821-823.

13. Berwick DM, Nolan TW, Whittington J: The triple aim: care, health, and cost. Health Aff 2008, 27(3):759-769.

14. Bovbjerg VE, Kingston MSJ: Program Impact Report: Oregon's living Well with Chronic Conditions. Oregon State University College of Health and Human Services; 2010. Retrieved May 11, 2013, from [http://public.health.oregon. gov/DiseasesConditions/ChronicDisease/LivingWell/Documents/Living\% 20Well\%20Program\%20Impact\%20Report\%20Final.pdf].

15. Page TF, Palmer RC: Cost analysis of chronic disease self-management programmes being delivered in South Florida. Health Educ J 2013. 10.1177/0017896912471047.

16. National Council on Aging: Chronic Disease Self-Management Education Integrated Services Delivery System Assessment Tool. Washington, DC; 2013. http://www.ncoa.org/improve-health/center-for-healthy-aging/contentlibrary/CDSME-Sustainability-Tool.pdf.

17. US Census Bureau: United States Census 2010. Washington, DC: US Department of Commerce; 2010. Retrieved May 15, 2013, from [http://www. census.gov/2010census/].

18. Ritter PL, Stewart AL, Kaymaz H, Sobel DS, Block DA, Lorig KR: Self-reports of health care utilization compared to provider records. J Clin Epidemiol 2001, 54(2):136-141.

doi:10.1186/1471-2458-13-1141

Cite this article as: Ahn et al.: The impact of chronic disease self-management programs: healthcare savings through a community-based intervention. BMC Public Health 2013 13:1141. 